Document downloaded from:

http://hdl.handle.net/10251/101832

This paper must be cited as:

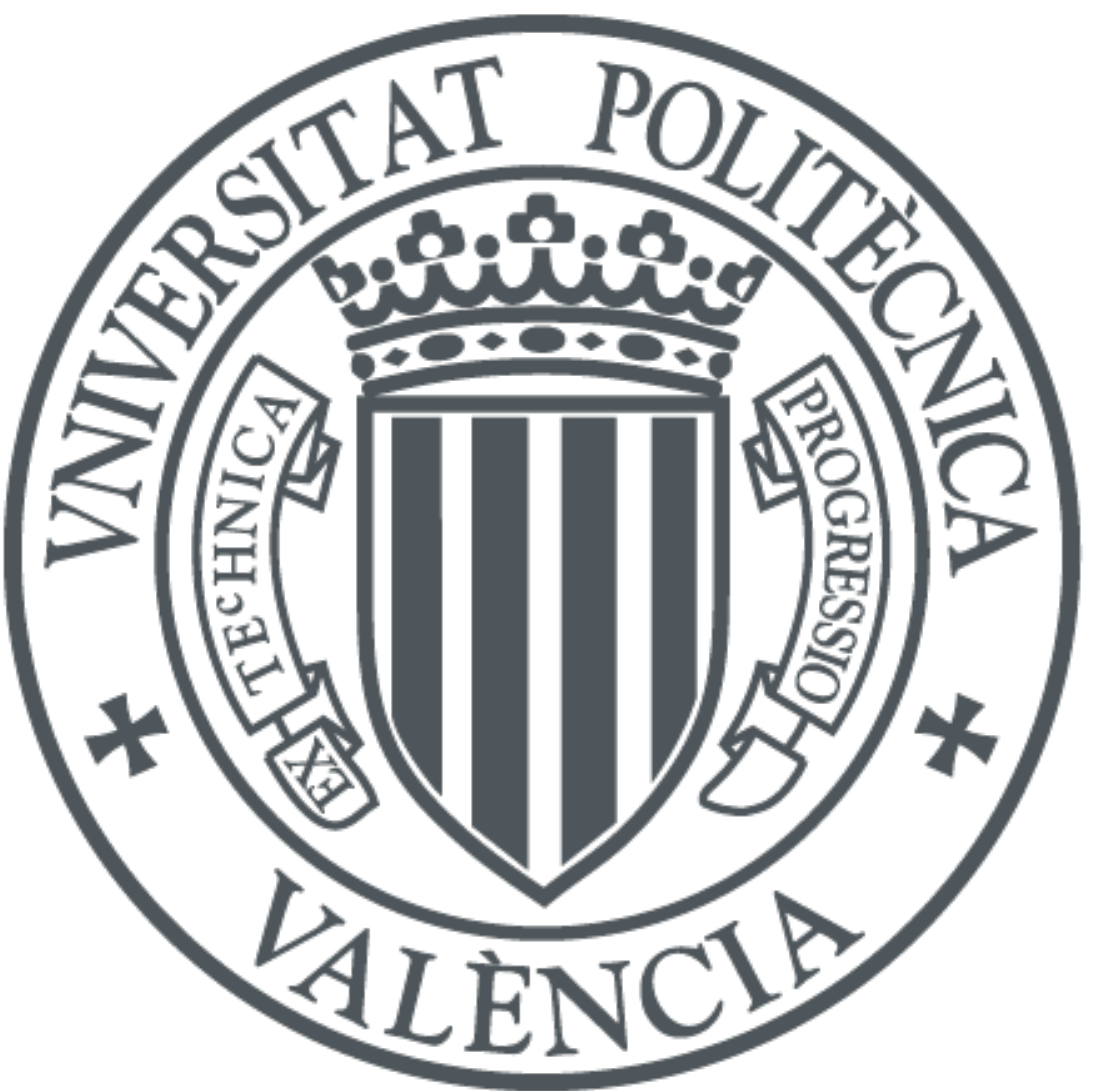

The final publication is available at

https://doi.org/10.1016/j.fss.2016.07.005

Copyright Elsevier

Additional Information 


\title{
On probabilistic $\varphi$-contractions in Menger spaces
}

\author{
Valentín Gregori ${ }^{1}$, Juan-José Miñana, Samuel Morillas \\ Instituto Universitario de Matemática Pura y Aplicada, Universitat Politècnica de \\ València, Camino de Vera s/n 46022 Valencia (SPAIN).
}

\begin{abstract}
In this note we show that the fixed point theorems given for Menger spaces by J. Jachymski [Theorem 1, J. Jachymski, On probabilistic $\varphi$-contractions on Menger spaces, Nonlinear Analysis 73 (2010) 2199-2203] and J.X. Fang [Theorem 3.1, J.X. Fang, On $\varphi$-contractions in probabilistic and fuzzy metric spaces, Fuzzy Sets and systems 267 (2015) 86-99] are equivalent.
\end{abstract}

Keywords: Fixed point, Menger space, probabilistic $\varphi$-contraction 2010 MSC: 54A40, 54D35, 54E50

\section{Introduction}

The problem of obtaining fixed point theorems for probabilistic $\varphi$-contractions (see Definition 2.1) in Menger spaces has been studied by different authors (see [1] and the references therein). In the former approaches to this topic the authors used conditions too much restrictive on the gauge function $\varphi$. Later, Jachymski in [4, Theorem 1] obtained a fixed point theorem for Menger spaces, defined for a continuous t-norm of Hadžić type, weakening the conditions on $\varphi$ which improved the applicability of these type of theorems. Recently, Fang [2, Theorem 3.1] has obtained a fixed point theorem that generalizes the Jachymski's theorem (in the context of Menger spaces) in two senses. In fact, Fang does not demand the condition of continuity for

\footnotetext{
Email addresses: vgregori@mat.upv.es (Valentín Gregori), juamiapr@upvnet.upv.es (Juan-José Miñana), smorillas@mat.upv.es (Samuel Morillas)

${ }^{1}$ Valentín Gregori acknowledges the support of Ministry of Economy and Competitiveness of Spain under Grant MTM2015-64373-P.
} 
the $t$-norm in his theorem, and, on the other hand, he demands a weaker condition on the gauge function $\varphi$. In this note, we prove that in the framework of Menger spaces a self-mapping $T$ of $X$ is a probabilistic $\varphi_{w}$-contraction in the sense of Fang if and only if it is a probabilistic $\psi$-contraction in the sense of Jachymski (Corollary 2.7). Consequently, the mentioned theorems are equivalent.

\section{Results}

Definition 2.1. Let $(X, F, *)$ be a Menger space. A mapping $T: X \rightarrow X$ is called a probabilistic $\varphi$-contraction, for $\varphi$, if it satisfies

$$
F_{T x, T y}(\varphi(t)) \geq F_{x, y}(t) \text { for all } x, y \in X \text { and } t>0,
$$

where $\varphi:[0, \infty[\rightarrow[0, \infty[$ is a gauge function satisfying certain conditions.

We will consider here three classes of gauge functions:

(i) $\Psi$ will denote the class of gauge functions $\psi$ satisfying:

$$
0<\psi(t)<t \text { and } \lim _{n \rightarrow \infty} \psi^{n}(t)=0 \text { for all } t>0 .
$$

(ii) $\Phi$ will denote the class of gauge functions $\varphi$ satisfying:

$$
\lim _{n \rightarrow \infty} \varphi^{n}(t)=0 \text { for all } t>0 .
$$

(iii) $\Phi_{w}$ will denote the class of gauge functions $\varphi_{w}$ satisfying:

$$
\text { for each } t>0 \text { there exists } r \geq t \text { such that } \lim _{n \rightarrow \infty} \varphi_{w}^{n}(r)=0 \text {. }
$$

Obviously, $\Psi \subset \Phi \subset \Phi_{w}$ and it is easy to verify that these inclusions are strict.

Recall that a $t$-norm $*$ is called of $H$-type (Hadžíc [3]) if the family of

functions $\left\{\prod^{m} * t: n \in \mathbb{N}\right\}$ is equicontinuous at $t=1$, where $\prod^{m} * t=t * \cdots * t$ $m$-times.

Remark 2.2. In [4, Theorem 1], J. Jachymski demands that * be a continuous t-norm of H-type. Nevertheless, the author does not use the continuity of $*$ in the proof of this theorem, but the continuity of $*$ at $(1,1)$. Now, the continuity of * at $(1,1)$ is fulfilled due to the equicontinuity of the family of functions $\left\{\prod^{m} * t: n \in \mathbb{N}\right\}$ at $t=1$, since $*$ is a $t$-norm of $H$-type. 
So, the Jachymski's theorem can be written, really, as follows.

Theorem 2.3 (Jachymski [4]). Let $(X, F, *)$ be a complete Menger space, where $*$ is a t-norm of $H$-type. If $T: X \rightarrow X$ is a probabilistic $\psi$-contraction, for $\psi \in \Psi$, then $T$ has a unique fixed point $x_{0} \in X$, and the sequence $\left\{T^{n}(x)\right\}$ converges to $x_{0}$ for all $x \in X$.

On the other hand the Fang's theorem is the next one.

Theorem 2.4 (Fang [2]). Let $(X, F, *)$ be a complete Menger space, where * is a t-norm of $H$-type. If $T: X \rightarrow X$ is a probabilistic $\varphi_{w}$-contraction, for $\varphi_{w} \in \Phi_{w}$, then $T$ has a unique fixed point $x_{0} \in X$, and the sequence $\left\{T^{n}(x)\right\}$ converges to $x_{0}$ for all $x \in X$.

Fang asserts in [2] that Theorem 2.4 is more general than Theorem 2.3. In the next, we will see that this assertion is not true showing that both theorems are equivalent. For it we will show that in the framework of Menger spaces the class of probabilistic $\varphi_{w}$-contractions, for some $\varphi_{w} \in \Phi_{w}$, coincides with the class of probabilistic $\psi$-contractions, for some $\psi \in \Psi$ (Corollary 2.7).

We start with the next theorem.

Theorem 2.5. Let $(X, F, *)$ be a Menger space and let $T: X \rightarrow X$ be a mapping. Then, $T$ is a probabilistic $\varphi_{w}$-contraction, for some $\varphi_{w} \in \Phi_{w}$, if and only if $T$ is a probabilistic $\varphi$-contraction, for some $\varphi \in \Phi$.

Proof Suppose that $T: X \rightarrow X$ is a probabilistic $\varphi_{w}$-contraction, for some $\varphi_{w} \in \Phi_{w}$. Then,

$$
F_{T x, T y}\left(\varphi_{w}(t)\right) \geq F_{x, y}(t) \text { for each } x, y \in X \text { and } t>0 .
$$

Denote $A:=\left\{t>0: \lim _{n \rightarrow \infty} \varphi_{w}^{n}(t) \neq 0\right\}$.

For each $t \in A$ we consider the set $B_{t}=\left\{r>t: \lim _{n \rightarrow \infty} \varphi_{w}^{n}(r)=0\right\}$, which is a non-empty set since $\varphi_{w} \in \Phi_{w}$. Then, by the Axiom of Choice, we can take an element $r_{t} \in B_{t}$, for each $t \in A$. Obviously, $r_{t} \notin A$, for each $t \in A$.

Now, we will construct a gauge function $\varphi \in \Phi$, such that $T$ is a probabilistic $\varphi$-contraction, for $\varphi$, as follows.

Define

$$
\varphi(t)= \begin{cases}\varphi_{w}(t), & \text { if } t \notin A \\ \varphi_{w}\left(r_{t}\right), & \text { if } t \in A\end{cases}
$$

Obviously, $\varphi$ is well-defined.

We will prove that $\lim _{n} \varphi^{n}(t)=0$ for all $t>0$ in three steps. 
(I) First we prove that if $t \notin A$ then $\varphi_{w}^{i}(t) \notin A$ for all $i \in \mathbb{N}$. Indeed, let $t \notin A$ and $i \in \mathbb{N}$. Then, $\lim _{n} \varphi_{w}^{n}\left(\varphi_{w}^{i}(t)\right)=\lim _{n} \varphi_{w}^{n+i}(t)=0$, and so $\varphi_{w}^{i}(t) \notin A$.

Now, we distinguish two cases:

(II) Suppose that $t \notin A$.

We will prove, by induction on $n$, that $\varphi^{n}(t)=\varphi_{w}^{n}(t)$ for all $n \in \mathbb{N}$. Indeed, $\varphi(t)=\varphi_{w}(t)$ since $t \notin A$. Suppose the assertion true for $n$. By induction hypothesis and by $(I)$ we have that

$$
\varphi^{n+1}(t)=\varphi\left(\varphi^{n}(t)\right)=\varphi\left(\varphi_{w}^{n}(t)\right)=\varphi_{w}\left(\varphi_{w}^{n}(t)\right)=\varphi_{w}^{n+1}(t) .
$$

Therefore, $\lim _{n} \varphi^{n}(t)=\lim _{n} \varphi_{w}^{n}(t)=0$.

(III) Suppose that $t \in A$.

We will prove, by induction on $n$, that $\varphi^{n}(t)=\varphi_{w}^{n}\left(r_{t}\right)$ for all $n \in \mathbb{N}$. The assertion is true for $n=1$ by definition of $\varphi$. Suppose the assertion true for $n$. By induction hypothesis and by $(I)$ we have that

$$
\varphi^{n+1}(t)=\varphi\left(\varphi^{n}(t)\right)=\varphi\left(\varphi_{w}^{n}\left(r_{t}\right)\right)=\varphi_{w}\left(\varphi_{w}^{n}(t)\right)=\varphi_{w}^{n+1}(t) .
$$

Therefore, $\lim _{n} \varphi^{n}(t)=\lim _{n} \varphi_{w}^{n}\left(r_{t}\right)=0$.

Thus, $\lim _{n \rightarrow \infty} \varphi^{n}(t)=0$ for each $t>0$, and then $\varphi \in \Phi$.

Finally, we will see that $T$ is a probabilistic $\varphi$-contraction for this $\varphi \in \Phi$. Let $x, y \in X$ and $t>0$. We distinguish two cases:

1. If $t \notin A$, then $F_{T x, T y}(\varphi(t))=F_{T x, T y}\left(\varphi_{w}(t)\right) \geq F_{x, y}(t)$.

2. If $t \in A$, then $F_{T x, T y}(\varphi(t))=F_{T x, T y}\left(\varphi_{w}\left(r_{t}\right)\right) \geq F_{x, y}\left(r_{t}\right) \geq F_{x, y}(t)$, since $r_{t}>t$ and $F_{x, y}$ is non-decreasing.

Therefore, $T$ is a probabilistic $\varphi$-contraction, for $\varphi \in \Phi$.

The converse is obvious since $\Phi \subset \Phi_{w}$.

To complete our purpose, we will show that the class of probabilistic $\varphi$-contractions, for $\varphi \in \Phi$, coincides with the class of probabilistic $\psi$ contractions, for $\psi \in \Psi$, in the next theorem.

Theorem 2.6. Let $(X, F, *)$ be a Menger space and let $T: X \rightarrow X$ be a mapping. Then, $T$ is a probabilistic $\varphi$-contraction, for some $\varphi \in \Phi$, if and only if $T$ is a probabilistic $\psi$-contraction, for some $\psi \in \Psi$. 
Proof Let $(X, F, *)$ be a Menger space and suppose that $T: X \rightarrow X$ is a probabilistic $\varphi$-contraction, for some $\varphi \in \Phi$. Then,

$$
F_{T x, T y}(\varphi(t)) \geq F_{x, y}(t) \text { for each } x, y \in X \text { and } t>0 \text {. }
$$

We claim that for all $t>0$ we can find $r>t$ such that $0<\varphi(r)<t$. Indeed, suppose there exists $t_{0}>0$ such that $\varphi(r) \geq t_{0}$ for each $r \geq t_{0}$. Then, for a fixed $r_{0} \geq t_{0}$ we have that $\varphi\left(r_{0}\right) \geq t_{0}$ and so $\varphi^{2}\left(r_{0}\right) \geq t_{0}$. Proceeding inductively on $n$ we obtain $\varphi^{n}\left(r_{0}\right) \geq t_{0}$ for each $n \in \mathbb{N}$ and so, $\lim _{n \rightarrow \infty} \varphi^{n}\left(r_{0}\right) \geq t_{0}$, a contradiction. Besides, $\varphi(r)>0$. Indeed, if $\varphi(r)=0$ we have that $0=F_{T x, T x}(\varphi(r)) \geq F_{x, x}(r)=1$, since $T$ is a probabilistic $\varphi$-contraction, a contradiction. Therefore, $0<\varphi(r)<t$.

Now, for each $t>0$ we consider the set $R_{t}=\{r \geq t: 0<\varphi(r)<t\}$, which is a non-empty set, as we have just seen.

For each $t>0$, we define

$$
\psi(t)=\frac{t+\inf \left\{\varphi(r): r \in R_{t}\right\}}{2} .
$$

The function $\psi$ is well-defined, since for each $r \in R_{t}$ we have that $\varphi(r)>0$ and clearly $\psi(t)>0$, for each $t>0$.

Next, we will see that $\psi \in \Psi$ in six steps.

(I) First, note that for each $t>0$ we have that $0<\psi(t)<t$. Indeed, by definition, for each $t>0$ we have that

$$
0<\frac{t}{2} \leq \psi(t)<\frac{t+t}{2}=t
$$

(II) The function $\psi$ is non-decreasing. Indeed, if we suppose that for some $0<s<t$ we have that $\psi(s)>\psi(t)$, then $\inf \left\{\varphi(r): r \in R_{s}\right\}>$ $\inf \left\{\varphi(r): r \in R_{t}\right\}$. Therefore, we can find $r_{t} \in R_{t}$ such that $0<$ $\varphi\left(r_{t}\right)<\inf \left\{\varphi(r): r \in R_{s}\right\}$. Taking into account that $\inf \{\varphi(r): r \in$ $\left.R_{s}\right\}<s$, then $r_{t} \in R_{s}$, since $r_{t} \geq t>s$ and $0<\varphi\left(r_{t}\right)<s$, and hence $\varphi\left(r_{t}\right)>\inf \left\{\varphi(r): r \in R_{s}\right\}$, a contradiction.

(III) The sequence $\left\{\psi^{n}(t)\right\}_{n}$ is strictly decreasing for each $t>0$. It is obvious, since for each $t>0$ we have that $0<\psi(t)<t$. Consequently:

(IV) $\left\{\psi^{n}(t)\right\}_{n}$ converges to the infimum of the sequence in $[0, \infty[$ with respect to the usual topology of $\mathbb{R}$.

(V) It is fulfilled that $\lim _{n} \psi^{n}(s) \leq \lim _{n} \psi^{n}(t)$ whenever $0<s<t$. This assertion is obvious since both limits exist and $\psi$ is non-decreasing. 
(VI) We will see that $\lim _{n} \psi^{n}(t)=0$ for each $t>0$.

Suppose that $\lim _{n} \psi^{n}\left(t_{0}\right)=a>0$ for some $t_{0}>0$. We assert that $\lim _{n} \psi^{n}(s) \geq a$ for each $s>a$. Indeed, if $s>a$ then we can find $n_{s} \in \mathbb{N}$ such that $\left.\psi^{i}\left(t_{0}\right) \in\right] a, s\left[\right.$ for all $i \geq n_{s}$. In particular $a<\psi^{n_{s}}\left(t_{0}\right)<s$. Then, by $(V)$ we have that $a=\lim _{n} \psi^{n}\left(\psi^{n_{s}}\left(t_{0}\right)\right) \leq \lim _{n} \psi^{n}(s)$.

We claim that there exists $t>a$ such that $\psi(t)<a$. Indeed, given $a>0$ we can find, as we have seen above, $r_{a}>a$ such that $0<\varphi\left(r_{a}\right)<a$. Now, note that for each $t \in] a, r_{a}$ [ we have that $r_{a} \in R_{t}$, since $r_{a} \geq t$ and $0<\varphi\left(r_{a}\right)<a<t$. Take $\delta>0$ such that $\delta<\min \left\{a-\varphi\left(r_{a}\right), r_{a}-a\right\}$. Then, for each $t \in] a, a+\delta[$ we have

$$
\psi(t) \leq \frac{t+\varphi\left(r_{a}\right)}{2}<\frac{a+\delta+a-\delta}{2}=a
$$

Choose $t>a$ such that $\psi(t)<a$. Then, $\lim _{n} \psi^{n}(t) \geq a$, since $t>a$. On the other hand, $\left\{\psi^{n}(t)\right\}_{n}$ is a strictly decreasing sequence with $\psi(t)<a$, a contradiction.

Therefore, $\lim _{n} \psi^{n}(t)=0$ for each $t>0$, and so $\psi \in \Psi$.

Finally we will see that $T$ is a probabilistic $\psi$-contraction for $\psi \in \Psi$.

First, note that for all $t>0$ we can find $r_{t} \in R_{t}$ such that $0<\varphi\left(r_{t}\right)<$ $\psi(t)$. Contrary, suppose that there exists $t_{0}>0$ such that for all $r \in R_{t_{0}}$ we have that $\varphi(r)>\psi\left(t_{0}\right)$. Then,

$$
\inf \left\{\varphi(r): r \in R_{t_{0}}\right\} \geq \psi\left(t_{0}\right)=\frac{t_{0}+\inf \left\{\varphi(r): r \in R_{t_{0}}\right\}}{2}
$$

and so $\inf \left\{\varphi(r): r \in R_{t_{0}}\right\} \geq t_{0}$. Now, by definition $t_{0}>\inf \left\{\varphi(r): r \in R_{t_{0}}\right\}$, a contradiction.

Let $x, y \in X$ and $t>0$. By the last paragraph we can take $r_{t} \in R_{t}$ such that $0<\varphi\left(r_{t}\right)<\psi(t)$. Then,

$$
F_{T x, T y}(\psi(t)) \geq F_{T x, T y}\left(\varphi\left(r_{t}\right)\right) \geq F_{x, y}\left(r_{t}\right) \geq F_{x, y}(t) .
$$

Therefore, $T$ is a probabilistic $\psi$-contraction, for $\psi \in \Psi$.

The converse is obvious since $\Psi \subset \Phi$.

As a consequence of these two last theorems we obtain the next corollary.

Corollary 2.7. Let $(X, F, *)$ be a Menger space and let $T: X \rightarrow X$ be a mapping. They are equivalent: 
(i) $T$ is a probabilistic $\psi$-contraction, for some $\psi \in \Psi$.

(ii) $T$ is a probabilistic $\varphi_{w}$-contraction, for some $\varphi_{w} \in \Phi_{w}$.

Consequently, Theorem 2.3 and Theorem 2.4 are equivalent.

Remark 2.8. The reader could find in this note a way of addressing the recent open question posed by Mihet in [6, Open question 1].

\section{Acknowledgements}

The authors are very grateful to the referees for their valuable suggestions.

[1] L. Cirić, Solving the Banach fixed point principle for nonlinear contractions in probabilistic metric spaces, Nonlinear Analysis 72 (2010) 2009-2018.

[2] J.X. Fang, On $\varphi$-contractions in probabilistic and fuzzy metric spaces, Fuzzy Sets and systems 267 (2015) 86-99.

[3] O. Hadžíc, Fixed point theorems for multivalued mappings in probabilistic metric spaces, Fuzzy Sets and Systems 88 (1997) 219-226.

[4] J. Jachymski, On probabilistic $\varphi$-contractions on Menger spaces, Nonlinear Analysis 73 (2010) 2199-2203.

[5] K. Menger, Statistical metrics, Pro. Natl. Acad. Sci. USA 28 (1942) 535-537.

[6] D. Mihet, C. Zaharia, On some classes of nonlinear contractions in probabilistic metric spaces

[7] B. Schweizer, A. Sklar, Statistical metric spaces, Pacific Journal of Mathematics 10 (1960) 314-334.

[8] B. Schweizer, A. Sklar, E.O. Thorp, The metrization of statistical metric spaces, Pacific Journal of Mathematics 10 (2) (1960) 673-675.

[9] B. Schweizer, A. Sklar, Probabilistic metric spaces, North Holland Series in Probability and Applied Mathematics, New York, Amsterdam, Oxford, 1983. 